Assurances et gestion des risques

Insurance and Risk Management

\title{
Analyse régulationniste des incertitudes : de l'assurance au principe de précaution
}

\section{Maria El Ghezaoui}

Volume 86, numéro 1-2, juin 2019

URI : https://id.erudit.org/iderudit/1062463ar

DOI : https://doi.org/10.7202/1062463ar

Aller au sommaire du numéro

\section{Éditeur(s)}

Faculté des sciences de l'administration, Université Laval

\section{ISSN}

1705-7299 (imprimé)

2371-4913 (numérique)

Découvrir la revue

\section{Citer cet article}

El Ghezaoui, M. (2019). Analyse régulationniste des incertitudes : de l'assurance au principe de précaution. Assurances et gestion des risques / Insurance and Risk Management, 86(1-2), 31-54. https://doi.org/10.7202/1062463ar

\section{Résumé de l'article}

Cet article analyse le statut du principe de précaution comme un principe de gestion de l'incertitude scientifique. Depuis quelques années, cette nouvelle référence normative est apparue dans le champ de l'environnement. Il a commencé à être reconnu par le droit international et par le droit interne. Ce texte présente les fondements du principe de précaution et discute ses limites majeures, tant juridiques que pratiques. Il met en avant certains avantages qu'il y aurait à développer des analyses, incluant plus de transparence dans les décisions de prévention et une meilleure évaluation de leur efficacité. Il suggère que les pratiques courantes de gestion des risques, assurance, ne sont pas toujours efficaces. Il explique la tension qui existe entre un système de prévention plus efficace et un système plus équitable. Il aborde les questions de prise en compte du long terme, de l'incertitude scientifique et des perceptions subjectives du risque par les assurances. Dans l'univers scientifiquement controversé, le principe n'a pas vocation à réduire l'incertitude mais au contraire à la porter comme question vivante dans la société. L'enjeu est l'invention de nouvelles procédures collectives dans le cadre desquelles les acteurs sociaux seront amenés à faire face de façon explicite et raisonnable aux situations risquées Godard (2000) et Triech (2001 et 2005).
Tous droits réservés (C) Faculté des sciences de l'administration, Université Laval, 2019
Ce document est protégé par la loi sur le droit d'auteur. L’utilisation des services d'Érudit (y compris la reproduction) est assujettie à sa politique d'utilisation que vous pouvez consulter en ligne.

https://apropos.erudit.org/fr/usagers/politique-dutilisation/ 


\title{
ANALYSE RÉGULATIONNISTE DES INCERTITUDES: DE L'ASSURANCE AU PRINCIPE DE PRÉCAUTION
}

\author{
Maria EL GHEZAOUI ${ }^{1}$
}

\section{RÉSUMÉ}

Cet article analyse le statut du principe de précaution comme un principe de gestion de l'incertitude scientifique. Depuis quelques années, cette nouvelle référence normative est apparue dans le champ de l'environnement. Il a commencé à être reconnu par le droit international et par le droit interne. Ce texte présente les fondements du principe de précaution et discute ses limites majeures, tant juridiques que pratiques. Il met en avant certains avantages qu'il y aurait à développer des analyses, incluant plus de transparence dans les décisions de prévention et une meilleure évaluation de leur efficacité. Il suggère que les pratiques courantes de gestion des risques, assurance, ne sont pas toujours efficaces. Il explique la tension qui existe entre un système de prévention plus efficace et un système plus équitable. Il aborde les questions de prise en compte du long terme, de l'incertitude scientifique et des perceptions subjectives du risque par les assurances. Dans l'univers scientifiquement controversé, le principe n'a pas vocation à réduire l'incertitude mais au contraire à la porter comme question vivante dans la société. L'enjeu est l'invention de nouvelles procédures collectives dans le cadre desquelles les acteurs sociaux seront amenés à faire face de façon explicite et raisonnable aux situations risquées Godard (2000) et Triech (2001 et 2005).

\section{ABSTRACT}

The purpose of this paper is to analyze the status of the precautionary principle of scientific and social controversies. This new normative reference has emerged in the recent years on the environmental scene. It is becoming acknowledged by international law and even by some national laws. This paper presents the foundations of the precautionary principle and discusses its main limits, either legal or practical ones. It puts forward some of the advantages from developing it further, including more decision transparency and a better efficiency assessment. 
It suggests that current practices of risk management and insurance are not always efficient. It explains that there are tensions between the efficiency and equity of prevention systems. It addresses the question of how to deal with the long run, with scientific uncertainties and with subjective risk perceptions by classic insurance. In a controversial scientific environment, the principle cannot be used as a tool for reducing uncertainty but as a way to elicit new collective procedures akin to precaution. The precautionary principle is there to lead social agents to explicitly address risk situations Godard (2000) and Triech (2001 et 2005).

\section{INTRODUCTION}

À l'heure actuelle, la mondialisation est indissociable de la multiplication et du croisement des crises et des risques qui dépassent les capacités d'anticipation des chercheurs et d'action des États quel que soit le niveau de développement de leur pays. Les risques, à l'image des catastrophes naturelles, se croisent et se cumulent. Baverez (2011) distingue dans ce sens: les risques de nature stratégiques avec les rivalités de puissance entre les pays; ceux de nature politique avec la montée du populisme dans les pays développés comme par exemple "le printemps arabe» qui aspire à la liberté et accompagne le développement dans le monde émergent; les risques asymétriques avec les États effondrés et le terrorisme; ceux de nature systémique qui découlent du fonctionnement des marchés financiers (la crise mondiale) sans négliger ceux qui sont liés à l'environnement, à la propagation des épidémies et à la technologie (la marée noire du golfe du Mexique, les accidents nucléaires, le génie génétique). Notre époque a donc redécouvert la notion de catastrophe et du risque.

Dans cette perspective, la conception traditionnelle des assurances s'enracine dans un apparent paradoxe entre le changement d'échelle des drames et la répartition financière difficile des assurances. Dans ce contexte, comment peut-on compenser par une valeur monétaire la pollution génétique, la pollution environnementale des sols ou l'érosion lente et irrémédiable de la biodiversité? Par conséquent, il serait erroné d'envisager les risques uniquement sous l'angle technicofinancier. Par exemple, les risques indéfinissables et imprévisibles liés aux organismes modifiés posent des problèmes cruciaux aussi bien aux assurés qu'aux assureurs comme on le verra dans ce qui suit.

Au-delà des conséquences financières potentielles qui peuvent être élevées, voire incalculables, la logique nécessite de prendre en considération la perte immatérielle liée au risque qui reste encore 
incommensurable (Lagadec, 1988). Envisagée comme un tout, la prise en compte des aspects socioculturels dans le calcul des assurances devient tout aussi cruciale que les aspects techniques. Il est important de souligner dans ce sens que le tout joue un rôle déterminant dans la nouvelle perception de ces nouveaux risques que l'humanité se crée elle-même. Arrivés à un point avancé dans les recherches en assurance, les chercheurs se trouvaient à une impasse. Ceci est causé par la part d'irréversibilité persistante, le risque dépasse les parties liées par le contrat d'assurance qui concerne désormais tous les acteurs et toutes les institutions.

Une fois admise l'existence des limites dans les assurances, la logique est de se tourner vers une autre notion de la responsabilité. Cette dernière mérite assurément que l'on s'y attarde même si sa mise en vigueur manifeste une certaine réticence à son égard. Il s'agit principalement du principe de précaution qui s'inscrit initialement dans les chartes de l'environnement et dont la valeur est constitutionnelle dans la majorité des pays. Ce principe est pourtant relativement moins fort dans les autres domaines. L'objectif était de se doter d'une nouvelle stratégie en matière de réglementation sur les substances chimiques et d'assurer un niveau élevé de protection de la santé et de l'environnement. Cela en améliorant la connaissance, l'information et en accélérant les procédures de gestion de risques imprévisibles. À cet effet, Daemen et Georgelaan (2013) ont soulevé que l'impact sur l'assurance est positif et qu'une meilleure connaissance des risques ne pouvant que favoriser l'assurabilité de ceux-ci.

Ce constat, nous renvoie à nous poser les questions suivantes : Qu'est-ce que le principe de précaution? S'agit-il d'un frein à la décision et au développement des assurances, d'un appel à un renforcement des exigences de sûreté et d'assurance, d'un levier de démocratisation des choix collectifs et d'assurance à caractère social? On voudrait répondre par l'affirmative à l'une et l'autre de ces questions. Nous proposons alors de se pencher sur des questions familières au principe de précaution comme le réchauffement climatique, les dangers des champs électromagnétiques pour démonter son importance. Dans un premier temps, le but de cet article est de contribuer à une meilleure compréhension des problèmes auxquels le principe de précaution est censé répondre avant de passer à dévoiler les difficultés qu'il engendre lui-même dans sa mise en ouvre en tant qu'élément d'assurance. Ainsi la première partie sera consacrée à la présentation des dimensions les plus caractéristiques de la notion de risque et de la relation risque-assurance usuelle. Nous plongerons dans la confection du système assurantiel pré-moderne pour arriver aux 
mécanismes utilisés actuellement. Dans un deuxième temps, nous présenterons les difficultés à recourir au principe de précaution en tant qu'illustration d'une possible nouvelle articulation de la relation incertitude-assurance.

\section{DE LA RESPONSABILITÉ CIVILE DES ASSURANCES À L'ÉTHIQUE DE LA RESPONSABILITÉ}

Ces dernières années, les débats se sont centrés sur les implications des nouveaux risques sur les assurances et les difficultés des assureurs de les prendre en compte. C'est l'assurabilité des incertitudes qui est remise en question. L'intensité de ce débat confirme l'idée qu'il est impossible d'atteindre le risque zéro. Ici, il serait toutefois trompeur de poser qu'il est possible d'investir des sommes infinies dans la prévention des risques mal définis. En tout état de cause, ceci nous conduit à définir des priorités, aussi en matière de prévention, et même si ces risques peuvent affecter nos vies, notre santé, nos proches et notre environnement. Dans la mesure où ces risques sont très difficilement évaluables, l'hypothèse de départ la plus raisonnable consiste à identifier: quels risques sont importants et lesquels ne le sont pas. Surtout, nous devons calculer combien cela coûte de changer le niveau de risque supporté. Cela permet de se concentrer sur les risques que l'on peut éliminer pour une moindre dépense (Triech, 2005).

Il est important de souligner dans cette optique que la prise de conscience de l'irréversibilité des décisions et d'incertitude radicale constitue une étape importante dans la perception des risques et l'amélioration du contrat socio-économique des assurances. Sur la question de la précaution, qui est la plus favorable au développement de la relation irréversibilité-assurance, la littérature théorique et empirique nous apportent un certain nombre d'enseignements.

\subsection{L'assurance confrontée aux incertitudes radicales: quelles perspectives?}

Partant de la définition des probabilités qui repose sur la notion de risques connus et mesurables. Les probabilités, grâce à des coefficients de tendance, permettent d'évaluer des risques homogènes en fréquence potentielle et en coût avec les périmètres classiques de mutualisation, par marché et dans le temps. Dès lors, les compagnies d'assurances 
traduisent la réalisation d'un danger indéterminé en "risque» en suivant un processus par lequel elles parviennent à déterminer son occurrence en appliquant les méthodes probabilistes et statistiques et pour mettre une valeur monétaire au dommage qui pourrait survenir.

Il existe des millions de composés chimiques, des centaines de milliers étant utilisés par l'industrie dans le monde. Chaque année, entre mille et deux mille nouvelles substances apparaissent sans qu'il soit aujourd'hui matériellement possible d'en déterminer l'impact sur l'environnement, sur la santé publique et sur l'avenir de la planète en général. Il en est ainsi, par exemple, des relais de téléphones mobiles qui ont fait l'objet de rapports officiels divers ne concluant pas unanimement sur les conséquences des ondes électromagnétiques. Dans ce cas particulier, les incertitudes peuvent résider dans différents domaines. De même, la diffusion des organismes génétiquement modifiés (OGM) aux États-Unis découle du fait que les propriétés d'un gène ne peuvent pas être considérées isolément, mais doivent être considérées de manière contextuelle. Elles dépendent de leur environnement génétique et s'expriment en interrelation avec d'autres gènes. Nous pouvons donc constater que les technologies du vivant sont des processus complexes, mal connus, d'où une forte méconnaissance des risques. Il s'agit d'un processus indéterminé et son effet est toujours aléatoire. Searalini (2003) souligne que «la complexité cachée du vivant peut provoquer des interactions métaboliques inattendues". D'une manière générale, il n'est pas étonnant dès lors que dans l'appréhension des risques liés aux organismes génétiquement modifiés restent en grande partie hypothétique et incertaine.

Dès lors, les exemples sont assez multiples dans ce sens: les histoires de la "vache folle» ou de l'encéphalite spongiforme bovine en Grande Bretagne, du sang contaminé en France, de la "crise à la dioxine" en Belgique. On pourrait bien évidemment multiplier les exemples mon seulement en environnement et en santé publique mais plus généralement dans toutes les activités humaines dont l'issue est recouverte par un "voile d'ignorance".

La difficulté de repérer les influences du développement scientifique au niveau global, tient aussi à la possibilité de la multiplication des catastrophes technologiques. Ceci nous conduit à avoir un commencement de doute sur un phénomène donné qui est évalué par une autorité scientifique reconnue quant à la potentialité d'un danger grave même sans une certitude scientifique absolue. Encore une fois, ceci contribue à la fragilité des organisations socio-économiques y compris les assurances (OCDE, 2003). 
Ce constat nous ramène à conclure que la démarche d'indemnisation adoptée par les assurances ne peut plus être suivie. Le futur incertain est traduit aujourd'hui par une plus grande volatilité des statistiques. Ce qui conduit à un gonflement des coûts et la réduction de la possibilité des engagements "long terme» des assureurs. Hélas, l'absence de probabilités rend le risque inassurable par l'industrie classique de l'assurance.

La difficulté de prendre ces nouveaux risques conduit les assureurs à être contraints de s'adapter pour pérenniser leur activité. Ils se trouvent face à l'apparition des risques auxquels ils ne peuvent plus offrir une couverture adéquate. Un Livre blanc de la Fédération française des sociétés d'assurances questionne la possibilité d'assurer les risques liés aux OGM. Ne pouvant plus garantir la prise en charge de toutes les conséquences des irréversibilités et des incertitudes, les assurances se trouvent dans une impasse devant la menace émanant des plantes transgéniques (Marie, 2014).

Cette situation est souvent considérée comme problématique, ne se réduisant pas à la seule responsabilité des assurances, mais cherchant à articuler l'ensemble des institutions. Alors qu'aucun des essais des OGM au champ libre n'est pas couvert par l'assurance par tout dans le monde, nous pouvons prendre dans ce sens l'exemple du rapport, élaboré sous l'égide du Ministère français de l'écologie et du développement durable, qui recommande d'inscrire dans la loi «l'obligation d'assurance pour passer du stade des essais confinés à celui des essais en plein champ" (November et November, 2004). En outre, cette illustration n'est pas isolée, les auteurs du rapport prévoient «la constitution d'un fond de solidarité par les opérateurs du secteur des biotechnologies» pour appuyer et aider le secteur d'assurance.

Toujours dans la même logique contractuelle ex-post, les assureurs insistent sur le fait que pour garantir un minimum d'assurance aux risques dont la fréquence de réalisation n'est pas connue, diverses conditions préalables doivent être remplies: en premier temps la quantification du risque et en deuxième temps l'évaluation des coûts prévisibles. Autrement dit, sous la pression concurrentielle, les assurances insistent sur les notions de "mesurabilité" et "prévisibilité" des risques pour qu'elles puissent les appréhender et les assurer. En revanche, Simonnet (2015) assure que les conséquences fortuites, accidentelles de l'utilisation des résultats des recherches scientifiques sont assurables, sous réserve de l'existence d'un cadre juridique bien claire. 
Le cadre législatif est pauvrement adapté aux problèmes qui peuvent surgir suite à des développements scientifiques. Par la logique actuarielle, l'absence d'une base légale qui prévoit l'attribution des responsabilités en cas d'accidents, ou sans précision de la période de prescription, fait que les assureurs ne peuvent pas agir. Actuellement face au problème inextricable que posent les éléments à grand risque, le cas par exemple des OGM, les États n'arrivent pas à trouver une parade satisfaisante pour harmoniser les points de vue de tous les acteurs sur les plans national et international.

Sous ces contraintes, notre point de vue est que la compréhension des mécanismes élémentaires du développement des assurances exige de recourir à l'aspect incertain des états de la nature. L'industrie de l'assurance avec ses outils classiques peut travailler avec légistes, juristes et décideurs publics pour faire du principe de précaution un facteur positif de progrès accepté avec une meilleure sécurité (Florin, 2004).

\subsection{Risque et Incertitude à l'ère de la réassurance}

Le lien entre risque-assurance comme déjà discuté auparavant se noue autour de l'inquiétude que provoquent les risques et l'aspiration à la sécurité qu'apporte l'assurance. En psychologie, le sens de la réassurance se manifeste sous forme d'un besoin ou d'un sentiment recherché, qui apparaît excessivement chez les individus en cas d'incertitude (Weinstock et Whisman, 2006). Selon Joiner et al. (1999), les individus qui sont incertains de leur environnement expriment un besoin de réassurance plus que les autres. Cette incertitude les conduit constamment à "des questions diagnostiques", notamment des questions de vérification qui visent la réassurance. Cette relation entre l'incertitude et la recherche permanente de réassurance peut appuyer nos propos dans cet article dans lequel nous nous intéressons à cette recherche de réassurance face à l'irréversibilité et l'incertitude (Hajtaieb et Yanhui, 2015).

La réassurance assume divers rôles importants dans l'économie: assureur en dernier ressort, elle fournit aux assureurs primaires la liquidité nécessaire à l'indemnisation des sinistres de gravité exceptionnelle (Thourot, 2016); elle mutualise la couverture des événements à grand risque, en apportant la capacité nécessaire pour la couverture de leur gravité. Elle peut être le promoteur de l'innovation en industrie d'assurance pour un développement responsable et durable. 
La réassurance est donc une forme compensatoire de l'incertitude. En effet, dans certains cas, les compagnies de réassurances exercent une pression sur leurs assurés afin qu'ils adoptent un comportement responsable permettant de réduire les risques dont les conséquences peuvent être irréversibles sur la société et l'environnement. En effet, une vaste réflexion qui s'est engagée pour déterminer si le changement climatique était susceptible d'affecter le bien-être, l'état de santé et la mortalité des populations.

Dans cette perspective la Compagnie de Réassurances Swiss Re de Zurich, par exemple, s'est adressée depuis 2001 aux grandes entreprises afin de les inciter à adopter des méthodes de production permettant de réduire leurs émissions de gaz à effet de serre, en menaçant les récalcitrants d'une augmentation considérable de leurs primes. Dans un cas pareil, le réassureur prend des mesures actives d'information ou de prévention; il peut en dernier recours refuser de couvrir certains risques. L'attitude interventionniste des assureurs est dictée par la nécessité (November et November, 2004).

En effet, les grandes assurances constatent que le coût des catastrophes a subi une forte hausse depuis plusieurs décennies. Cette évolution entraîne l'augmentation des sommes déboursées pour les indemnités qui entraîne les conséquences financières qui peuvent menacer leur solvabilité et provoquer même leur faillite. Par exemple, les coûts des catastrophes naturelles qui se sont succédés au troisième et quatrième trimestres 2018 ont sévèrement entaillé les profits des compagnies d'assurances. Par exemple Axa, le premier opérateur français en assurance et en réassurance, a vu son résultat net chuter de $66 \%$ (2,1 milliards d'euros) en partie à cause des indemnisations liées aux événements naturels. Celles-ci lui ont coûté deux milliards d'euros sur l'année 2018, sa filiale américaine a été particulièrement touchée. Les feux de forêt en Californie et l'ouragan Michael lui ont respectivement coûté 335 et 261 millions d'euros (Dumas, 2019).

La fréquence de répétition des catastrophes naturelles a tendance à augmenter et les coûts économiques suivent: «selon le rapport annuel sur les catastrophes naturelles dans le monde publié fin janvier 2019 par le courtier Aon, les pertes économiques se sont élevées à 225 milliards de dollars dans le monde en 2018, dont 90 milliards de dollars de dégâts assurés"(Dumas, 2019). Ceci laisse croire que, malgré la contribution de la réassurance dans l'industrie de l'assurance, les risques à grande échelle donnent du fil à retordre aux assureurs. Il s'avère ainsi qu'il est impossible de maîtriser parfaitement le risque 
ex-post, d'où le recours à la prévention et à la précaution. L'assureur a tout intérêt à ce que l'assuré tente de maîtriser au maximum ses risques assurables ex-ante, ce qui diminue les probabilités de réalisation, donc les primes, et ce qui bonifie le portefeuille assurable (Dumont, 2011).

\subsection{Un sens éthique à retrouver : vers un principe de précaution}

La racine latine du mot "précaution" renvoi au fait de prendre garde d'une menace plus diffuse, plus subjective. Depuis son apparition, le principe est utilisé en santé publique au début des années 90 dans l'affaire de la vache folle et l'application du principe a touché le domaine de l'environnement et des changements climatiques. Actuellement, le principe de précaution est de grand usage comme principe fondamental de la théorie de la décision et une nouvelle règle de comportement individuel face à l'incertitude. Une règle qui repose sur l'idée que chaque décideur doit s'efforcer d'éviter la dégradation de sa situation initiale, c'est-à-dire qu'il doit attendre pour améliorer ses connaissances dans un contexte où l'information future est susceptible de réduire l'incertitude radicale (Triech, 2001). On invoque l'application du principe de précaution dans les domaines les plus hétérogènes (climat, couche d'ozone, faune, flore, pêcheries, OGM, santé, alimentation, jusqu'aux conditions d'utilisation des armes sur les champs de bataille). Il est donc devenu une référence majeure qui trouve son exercice dans une situation d'incertitude scientifique.

Par ailleurs, l'attentisme n'est pas un comportement familier pour les économistes qui préfèrent étudier l'épargne. Néanmoins, différents travaux ont montré son importance dans les situations d'incertitude. Keynes (1936) dans ce sens l'avait évoqué dans sa théorie générale avec le motif de la spéculation. Les investisseurs qui anticipent une hausse des taux conservent de la liquidité et reportent leurs décisions d'investissement dans les titres financiers.

À la fin du $\mathrm{XX}^{\mathrm{e}}$ siècle, les économistes ont associé l'attentisme à la notion d'irréversibilité et de valeur d'option, attendre devenant un comportement susceptible d'être optimal. En effet, l'aspect séquentiel de la décision d'investir impose cette façon d'agir. C'est particulièrement important pour les problèmes d'investissement qui relèvent de politiques à très long terme qui sont susceptibles d'être révisées à la lumière des progrès de la connaissance scientifique des investisseurs et de la technique des univers (Duong, 2005). 
De ce fait, pour traiter la précaution, il faut donc tenir compte de différentes faces de l'ignorance y compris l'incertitude non spécifique et l'incomplétude provenant des autres sources (surprises possibles, controverses, tabous, ...). Selon Barrieu et Sinclair-Desgagné (2003), c'est une politique nécessairement tournée vers l'avenir tenant compte de notre fragilité, des limitations de nos ressources, des équilibres vitaux et l'évolution de l'incertitude avec le temps. L'irréversibilité des nouveaux risques technologiques, amplement discutée en premier chapitre, nous y convoque de manière urgente. Le principe de précaution émerge à ce stade de la réflexion, compte tenu de la situation de couverture restreinte par les assurances des dommages liés aux incertitudes radicales.

Hunyadi (2003) précise que l'insécurité toujours plus élevée ressentie par la population au sujet des risques possibles (dont la probabilité peut être cependant minime) contraste avec l'affaiblissement de l'institution de l'assurance. Comme le souligne le professeur de philosophie Mark Hunyadi de l'université Laval, il convient de remarquer que le principe de précaution, tel qu'il est mis en pratique, ne s'applique pas seulement aux risques dont la réalisation est incertaine au vu des connaissances scientifiques d'une époque donnée, mais aussi les risques avérés, ceux dont l'existence est démontrée empiriquement. Par conséquent, le principe de précaution appelle donc non seulement une attitude de précaution, mais aussi une attitude de prudence et de prévention $^{2}$. Un spécialiste de la santé publique voit à travers le "principe de précaution ${ }^{3}$ " une reprise de la démarche épidémiologique classique face au risque: confrontée à une maladie dont les causes ne sont pas élucidées, la prévention s'organise, sans attendre des certitudes, à partir de l'analyse des facteurs de risques qui ne sont pas des causes (Abenhaim, 1999).

Afin de bien concrétiser nos propos dans ce chapitre, nous introduisons le point de vue juridique. En droit, l'étude des principes exige que soient triés dans l'amas actuel des énoncés à prétention principielle, ceux qui constituent réellement des principes.

$\mathrm{La}$ "précaution" a requit ce caractère de "principe" pour au moins trois raisons. La première est essentiellement d'ordre pratique. C'est dans le cadre de différents problèmes récents relatifs à des incertitudes radicales et d'un retentissement important que l'on a vu évoquer de façon croissante ${ }^{4}$. La deuxième raison est d'ordre politique et scientifique. Le "principe de précaution" modifie les rapports entre décision politique et fondement scientifique de cette décision. La troisième raison est d'ordre juridique. On retrouve aujourd'hui de nombreux 
textes à caractère contraignant ou non relatifs au "principe de précaution" qu'il importe de bien cerner car ils fournissent des références telles que les lois sur l'air et sur l'eau (Yombatina, 2010). Cette configuration juridique nous permet de démonter l'importance du principe de précaution comme attitude à entreprendre lorsqu'il s'agit d'un univers d'incertitude radicale.

Pour compléter notre analyse, nous proposons de préciser les aspects multidimensionnels du principe de précaution qui peuvent devenir centrales dans les recherches sur les assurances. En ce sens, la précaution associe à la fois la prévention et la protection pour en limiter les effets. Elle ajoute aussi l'acceptabilité sociale et économique du risque. Le principe de précaution instaure non seulement un état de vigilance continuel pour guetter l'arrivée de nouveaux risques collectifs qui pourraient menacer, le cas échéant, la sécurité des personnes, leur santé et de l'environnement; mais il impose aussi un dialogue permanent avec toutes les parties concernées pour adapter les limites de la protection assurée en fonction de l'évolution rapide et mouvante des connaissances scientifiques et techniques. Tout comme pour les mesures de l'auto-assurance, l'observation ex-ante des comportements permet de remonter au consentement et à payer pour diminuer la probabilité de risque. Ainsi, il offre de "nouveaux repères dont les acteurs sociaux se saisissent pour réorganiser le jeu de la décision collective" (Godard, 2000).

Le principe de précaution est d'ordre contingent et porte sur les intentions des individus d'avoir des comportements spécifiés. On est ici dans le cas de l'approche de l'assurance basée sur l'évaluation des risques en amont qui permettrait une meilleure préparation de la prise de décision dans l'incertitude ${ }^{5}$. L'application du principe de précaution amènera vraisemblablement les assureurs à déplacer l'accent qu'ils mettent encore sur les conséquences de la réalisation des risques, vers l'anticipation des risques majeurs (en mettant en place une sorte de "early warning system"). Selon November et November (2004), les assurances deviendraient alors des partenaires proactives dans l'élaboration des hypothèses lors de la mise en œuvre du principe de précaution.

Ainsi, à l'encontre de l'assurance qui porte sur des risques exogènes, la démarche de précaution concerne des risques endogènes, c'est-à-dire dont nous savons que nous en sommes les auteurs, les causes, que nous en sommes à l'origine. Ainsi pourrait-on parler de la précaution comme l'assurance des risques endogènes. Sous cette dernière hypothèse, les assureurs seront bien sensibilisés aux risques majeurs. 
En général, dans la situation d'incertitude, la conception de la responsabilité à l'égard des dommages se transforme. La seule responsabilité civile qui impute individuellement une cause ou une faute à l'origine d'une catastrophe quitte à s'orienter de plus en plus vers un régime de responsabilité collective ${ }^{6}$. En d'autres termes, on passe d'une attribution individuelle de la responsabilité à une socialisation de la responsabilité des dommages.

\section{LES DIFFICULTÉS DE LA MISE EN OEUVRE DU PRINCIPE DE PRÉCAUTION}

Le déclenchement du débat sur le rôle du principe de la précaution ne suffit pas pour adopter une démarche d'assurance socialisante. Il faut aussi le mettre en œuvre. Toutefois, sa mise en œuvre interpelle tous les acteurs, scientifiques, décideurs politiques et économiques, membres de la société civile, pour les interroger sur la nature et le degré des risques que la communauté est disposée à accepter et à assumer.

En dépit de cette reconnaissance, Nous pouvons distinguer deux limites essentielles à sa mise en ouvre: il s'agit d'une part de son caractère implicite dans le droit et d'autre part la faible implication des acteurs.

\subsection{Le «principe de précaution» a-t-il une valeur normative autonome dans les ordres juridiques?}

La fonction de prévention n'est pas si nouvelle dans les ordres juridiques nationaux et internationaux. Elle peut prendre deux formes distinctes: une fonction classique de dissuasion qui vise la prévention des fautes et qui est fondée sur la responsabilité civile des assurances classiques et une fonction nouvelle celle de précaution comme déjà vue auparavant fondée sur l'anticipation de dommages graves et irréversibles.

Dans cette alignée, transposé dans le domaine du droit civil, le principe de précaution pourrait être considéré comme analogue à une règle d'extrême prudence. De ce fait, la précaution est traditionnellement reconnue par la jurisprudence comme l'équivalent de la prudence et son absence comme une faute d'abstention (Daemen et Georgelaan, 2013). Or, il semble ici supposer, en se référant à la définition du 
principe de précaution, que la prévention n'est pas dynamique. Dès lors, en matière du décalage temporel, ce n'est pas la prévention qu'il faut développer mais en contrepartie c'est le principe de précaution qui doit regagner de l'intérêt. Malgré l'importance reconnue au principe de précaution, les textes ne disent rien dans ce sens. Une cause préliminaire à cette limite concerne peut-être la pluralité des définitions de la précaution selon les pays et selon les domaines d'application. Nous rejoignons dans ce sens, Ewald $(2012)^{7}$, lorsqu'il dit qu' «il existe une multiplicité de principes de précaution. C'est la première des raisons pour laquelle il faudrait y être opposé».

En droit, il est largement retenu qu'un principe doit avoir, nécessairement, un caractère normatif comme déjà vue auparavant. Cette nécessité telle qu'elle est spécifiée, nous conduit à nous poser les questions suivantes concernant le statut juridique du principe de précaution: De quels principes parle-t-on? S'agit-il d'une règle de droit à part entière? Ou d'un "principe politique» destiné à guider l'action législative et réglementaire? Est-il suffisamment précis et cohérent pour que l'on puisse en déduire des conséquences juridiques?

Les travaux scientifiques sur le principe de précaution, bien que plus fragiles de par leur spécification, sont susceptibles d'apporter quelques réponses à nos questions (Gollier et Treich 2014). La justification de la précaution selon les auteurs se fonde ainsi sur l'étude du comportement individuel en situation d'incertitude par la modélisation et en utilisant les données de terrain. Ces données proviennent de l'observation des décisions individuelles sur les différents marchés les plus risqués (par exemple la bourse, la demande d'assurance des risques de haut niveau de gravité). Le cas le plus empirique du principe de précaution sous forme d'une valeur d'option est celui de Nordhaus (1994) qui a développé une analyse de Monte Carlo qui a calculé le timing optimal de réduction des émissions de $\mathrm{CO} 2$, compte tenu des hypothèses différentes sur la date à laquelle tous les paramètres du modèle DICE seront effectivement connus.

Aujourd'hui, l'enjeu est de savoir si le principe de précaution a un impact sur la responsabilité pénale et civile des particuliers, des entreprises ou de l'Etat même. Le cas des faucheurs volontaires de plantes OGM est considéré comme exemplaire en matière de responsabilité pénale (Bernard et al, 2010). Ils ont revendiqué le principe de précaution comme un principe d'immunité pénale. Dans la mesure où l'Etat ne prenait pas de dispositions pour transposer les directives européennes, ils faisaient, selon eux, ce que l'Etat aurait dû faire. Mais la 
Cour de Cassation non convaincue a rejeté leur revendication. Cet exemple explique pour une large part l'intensité juridique du principe qui varie en fonction de l'ordre juridique dans lequel il est repris: international, communautaire ou national.

À l'appui de ce constat, nous pouvons remarquer le faible usage du principe en matière de responsabilité civile (Camproux-Duffrene et Muller-Curzydlo, 2011). En effet le débat n'est pas encore tranché. Le principe de précaution semble évoluer au gré des libres interprétations des juges, sans un cadre juridique bien claire, créant ainsi une insécurité juridique. Dans cette perspective nous pouvons citer la confirmation de la Cour d'Appel de Versailles de la décision des juges de première instance qui avaient ordonné le démantèlement d'une antenne Bouygues Télécom au nom du principe de précaution et l'indemnisation des plaignants. De ce fait, les juges ont reconnu alors qu'une situation d'incertitude sur le risque couru pouvait engendrer un stress pour une personne exposée, lui donnant le droit de faire cesser le trouble. Au contraire, la cour de Cassation pour la même affaire a estimé que le principe de précaution ne modifiait pas les règles de responsabilité civile et que le dommage devait être certain (FantoniQuinton et Saison-Demars, 2016).

Défenseurs d'un ordre social supposé, la Cour de Cassation Française a encore refusé d'appliquer le principe. Un éleveur demandait à être indemnisé pour les problèmes de santé dont était victime son élevage porcin et bovin, causés, selon lui, par une ligne de haute tension (Dufumier, 2017). La Cour a jugé que sa demande n'était pas recevable, en mettant en avant l'absence de certitude scientifique sur la nocivité de l'exposition aux champs électromagnétiques. "La charte de l'environnement et le principe de précaution ne remettent pas en cause les règles selon lesquelles il appartient à celui qui sollicite l'indemnisation du dommage à l'encontre du titulaire de la servitude d'établir que ce préjudice est la conséquence directe et certaine de celui-ci» (Forest, 2011).

En général malgré l'arsenal important des traités et des conventions, le "principe de précaution" reste aussi sans application. Par ailleurs il est souvent évoqué à plusieurs reprises devant les juridictions internationales mais sans suivi efficace. Dans ce cas prenons l'exemple de la Nouvelle-Zelande lorsqu'elle a voulu s'opposer à la France devant la Cour Internationale de justice, lors de la reprise des essais nucléaires en 1995. La France a dû procéder à une évaluation de l'impact de ces essais nucléaires sur l'environnement avant de les entreprendre et démontrer qu'elles étaient sans risque. La Cour ne s'est pas prononcée 
sur la question. Même chose pour l'affaire d'installations hydrauliques sur le Danube opposant la Hongrie à la Slovaquie, la Cour a écarté l'argumentation hongroise, considérant que le péril dont se plaignait la Hongrie était à la fois trop incertain et trop lointain (Yombatina, 2010).

Devant les juridictions de l'Organisation Mondiale du Commerce (OMC), le "principe de précaution" semble promis à un bel avenir: il s'agit d'arbitrer le conflit entre le principe de la liberté des échanges, de la propagation du capitalisme ravage et les mesures prises par chaque État pour protéger la santé de sa population ou son environnement. Mais étant donné ces efforts, nous constatons que certains pays refusent d'appliquer ce principe. Nous donnons ici l'exemple de l'affaire du bouf aux hormones, quand les États-Unis considéraient que le "principe de précaution" ne pouvait pas revêtir le statut de règle coutumière internationale; il fut suggéré qu'il s'agissait simplement d'une approche de prudence que d'un principe (Martineau, 2013).

Mais, dans la jurisprudence de la Cour de justice de l'Union européenne le principe est pris comme catégorique, prenons ici l'exemple de l'arrêt sur la validité de la décision de la Commission interdisant l'exportation du bouf du Royaume-Uni pour limiter le risque de transmission (Hémon, 2015).

À proprement parler, souvent cette insécurité juridique est due au cadre des contentieux de légalité, tant des juridictions communautaires que des juridictions administratives nationales et internationales. En effet le paradoxe coexistant entre l'exercice de légalité interne et le respect de légalité externe met les juges sous contrôle plus étendu (Audouy, 2015).

Ainsi, dans l'affaire de la vache folle, la Cour de justice de l'Union européenne n'a pas tardée à utiliser le "principe de précaution" qui est bien clair et inscrit à l'article 174, paragraphe 2 du Traité (GiudicelliDelage, Manacorda et d'Ambrosio, 2016).

Dans la même veine, le Conseil d'Etat français a recouru à plusieurs reprises au "principe de précaution" tantôt pour valider des normes de protection de la santé, tantôt pour suspendre un arrêté autorisant la commercialisation du maïs transgénique. Néanmoins, la Cour de justice de l'Union européenne a jugé illégale la position de la France. Sous argument que les textes européens soutiennent la liberté de la recherche et la liberté d'entreprendre, qui ne peuvent pas être suspendues par précaution et, dans certains cas, que sur la base d'une preuve scientifique qu'il y a un danger (Ewald, 2012). 
Au Maroc, le principe de précaution n'a pas fait débat, par rapport à sa reconnaissance juridique dans le monde. Il est formulé en Allemagne dès la fin des années 1960. Et aux États-Unis, la création de l'Agence de protection de l'environnement, qui a des pouvoirs de contrainte très forts, date de 1969. Cependant au Maroc aucune plainte n'est portée même si les industriels polluants empoisonnent la vie des millions des habitants et dégradent la fertilité des terres agricoles, surtout dans la région Casablanca.

Au regard de ce qui précède, la mise en vigueur du principe de la précaution n'a qu'un caractère provisoire. Ceci implique qu'un effort non négligeable reste à déployer pour obtenir ou générer les données scientifiques nécessaires. En effet, en procédant de la sorte, les juridictions communautaires, nationales et internationales n'ont qu'une marge d'appréciation plus au moins importante lorsqu'elles adoptent des mesures de police administrative dans un contexte d'incertitude scientifique.

En plus de cette insécurité juridique, dont souffre le principe, il fait peser un risque de dysfonctionnement sur l'assurance de responsabilité civile. Car, pour fixer un tarif d'assurance, l'assureur doit pouvoir mesurer ses engagements et les risques qu'il accepte de couvrir.

Consciente de cette problématique, une solution partielle de réassurance est donnée par le modèle français. La France a adopté une résolution sur la mise en ouvre du principe de précaution, en préconisant en particulier de mettre en place une procédure d'identification de l'émergence de nouveaux risques pour l'environnement, la santé publique et la sécurité alimentaire et bientôt financière. Une initiative qui pourrait contribuer à améliorer la protection des citoyens et des assurés. Cette résolution adoptée concerne aussi, la perception par les entreprises d'une évolution du droit applicable en matière de responsabilité civile, renforçant leurs obligations.

Bien qu'il reste formulé en termes très généraux, conjonction de prudence et de vigilance appliquée aux activités de la vie moderne, le principe de précaution fait néanmoins son apparition dans le champ juridique et donc dans les décisions opérationnelles.

Toutefois, pour revêtir un caractère autonome, le principe de précaution doit remplir deux conditions: d'une part il doit être coulé dans un texte à portée normative et d'autre part il doit être formulé de manière suffisamment prescriptive. C'est au regard de cette double approche qu'il pourra s'imposer, tout au moins sur un plan théorique, dans les différents ordres et sphères de l'industrie d'assurance. Ceci 
nous renvoie à la question suivante: la négligence du fondement juridique du principe est dû à une obligation de concision ou simple oubli? Nous y reviendrons dans le chapitre suivant.

\subsection{Principe de précaution et responsabilité des opérateurs publics et privés}

Pour le second point, le principe de précaution est toujours un principe de responsabilité de l'Etat, les pouvoirs de police de l'Administration engageant sa responsabilité. Dans les exemples passés du sang contaminé ou de l'amiante, il a ainsi été jugé que l'Etat n'avait pas suffisamment fait pour protéger la population. Encore un autre exemple dans ce sens, dans le cas où il y a eu contamination d'une parcelle non OGM par des semences OGM, il est certainement difficile d'identifier à qui incombe la faute: au semencier, au négociant ou à l'agriculteur voisin? Le rapport adressé au Ministère français de l'écologie et du développement durable, propose d'instaurer un principe de responsabilité objective sans faute en matière de dissémination de OGM, tout en étant conscient que la détermination de la faute reviendra à l'expertise. Le rapport en question note enfin, que la responsabilité administrative qui incomberait à l'Etat qui délivre l'autorisation de dissémination d'une plante transgénique devrait être examinée sous l'angle du principe de précaution en l'état actuel du droit (Babisuaux et al, 2003).

Sur la base des exemples précédents, il apparaît que la mise en œuvre du principe est attribuée aux autorités publiques sans aucun doute, mais n'y a-t-il pas, associée inévitablement à cette morale publique, une responsabilité des opérateurs privés?

Ce principe, né du souci de préservation de l'environnement et auquel l'assurance s'efforce malgré tout d'échapper, détermine désormais de plus en plus les relations du droit et de la science avec l'ensemble des décisions opérationnelles publiques ou privées.

On l'aura bien compris à travers ce qui précède que la démarche de précaution, avec la dimension d'incertitude qui l'accompagne, impose d'anticiper, de prévenir, de prendre le devant, d'aller au-devant du risque et de la menace avant qu'elle ne se réalise (Yombatina, 2010). Cela change tout: non pas tant la volonté de réduire les risques que la manière d'y parvenir. Dans cette optique, le véritable problème sera de savoir si l'application du principe de précaution, à la charge des opérateurs, est compatible avec la reconnaissance de l'exonération pour risque de développement. Des conséquences juridiques et opérationnelles peuvent désormais en découler. Faut-il que l'assurance plonge 
dans ce contexte encore trouble d'un droit encore flou qui est celui du principe de précaution? Qu'impliquerait donc, au niveau économique, politique, juridique et à l'échelle à la fois nationale et internationale, le fait de mettre en ouvre le principe de précaution dans la gestion des risques?

C'est cette attitude de refus de prendre en compte la possibilité de réalisation totale d'un "scénario-catastrophe" qui peut être acquis comme une réponse préliminaire à nos questions. Baverez (2011) dénonce, à cet effet, que la mise du principe de précaution au centre des politiques nationales et internationales de gestion des risques est paralysante pour les États et les populations dans la mesure où ils s'interdisent de mettre en ouvre des plans d'actions efficaces en refusant de stipuler que des scénarios catastrophes puissent se réaliser.

Afin de compléter nos propos, il reste de mentionner que les chercheurs scientifiques reconnaissent que l'émergence de la problématique de précaution dans les débats sociétaux les conduit à s'interroger sur le sens et la portée de leurs activités. Ils font aussi part d'une certaine inquiétude quant à un usage effectif du principe par les autorités publiques en tant qu'outil de bonne gouvernance.

C'est une inquiétude partagée par les entreprises qui craignent d'être empêchées d'exercer leurs activités. Il s'ajoute aussi l'incapacité dans laquelle certaines entreprises se trouvent à s'assurer pour leurs activités potentiellement concernées par le principe de précaution, telle que l'industrie des biotechnologies où l'exercice du principe de précaution est fréquent. Il est de fait que les entreprises ne sont pas favorables à l'application du principe. Ce refus s'explique par leur perception de l'évolution du droit applicable en matière de responsabilité civile concernant le principe. Pour autant c'est bel et bien le droit appliqué qui dans ce sens peut renforcer les obligations d'entreprises au point, le cas échéant, de les dérouter quant à la définition même de ces obligations. "Que le principe de précaution soit édicté, fort bien, mais s'il n'est pas prolongé par une politique nouvelle en matière d'assurance, il sera soit funeste, soit caduc!»comme le signale le député Goasguen (2011).

Pour cette raison la volonté d'instaurer un tel principe dans le contexte actuel nécessite un basculement de l'imaginaire collectif et des pratiques sociales touchant à la science et à la technique vers une systématisation du doute et de la controverse. Si la foi dans les bienfaits de la science et du progrès technique n'a pas disparu, il reste qu'elle n'est plus constitutive du lien social au moins en ce qui concerne les 
perspectives d'un développement pour l'ensemble de l'humanité. La sécurité apportée à certains membres de la société n'empêche pas, en inverse, une large exclusion économique et sociale de se développer pour d'autres membres de la même société. En de nombreux cas, les erreurs de jugement et compromission tolérée entre des intérêts de santé publique et des intérêts industriels conduisent à des défaillances.

Ce paradoxe apparent de disparité entre les hommes s'accentue à cause du recul des institutions qui devaient apporter la sécurité. Souvent elles semblent débordées par le mouvement heurté des innovations et des pratiques hors normes. Pour ces diverses raisons, si bien qu'il a fallu que les États révisent d'urgence les règles d'engagement de la responsabilité pénale des agents publics pour des faits d'imprudence, de négligence ou de corruption.

La variante du principe de précaution est l'action adressée aux décideurs. En premier lieu, il est bien sûr destiné aux autorités publiques qui, dans l'incertitude, ont la responsabilité d'action pour autoriser ou interdire des produits, accorder l'ouverture ou la fermeture de sites d'exploitation par exemple.

Mais aujourd'hui, même si le débat reste ouvert, ce principe tend à s'appliquer aussi pour des décisions d'ordre privé et notamment pour les chefs d'entreprises. Dans ce cas un opérateur pourrait voir sa responsabilité engagée sur ce terrain, par exemple s'il ne retire pas un produit du marché à partir du moment où un doute existe quant à sa sécurité et cela avant même la réalisation d'un dommage. Par ailleurs, les décideurs privés sont juridiquement et politiquement obligés à faire un suivi de l'état des connaissances scientifiques après la mise sur le marché ou même après l'autorisation administrative pour les produits qui le nécessitent. Ainsi, dans le cas de la simple naissance d'un doute, l'opérateur devrait non seulement arrêter la fabrication mais rappeler tous les produits déjà mis en circulation (Florin, 2004).

Enfin, il convient donc de donner des définitions précises au contenu et à l'application du principe de précaution si son usage se dirige vers la responsabilité civile. Les critères retenus dans la sélection du niveau de risque acceptable et donc présentant une sécurité jugée suffisante varieront selon les situations. Ce sont là des jugements de valeur et de la responsabilité de définir ce qui est acceptable qui ne peuvent pas être assumés par les seuls experts scientifiques ou les pouvoirs publics, il faut associer dans ce processus aussi la société civile. 


\section{CONCLUSION}

En conclusion, le principe de précaution est un nouveau concept juridique qui mérite une réflexion profonde et qui a trouvé un écho favorable au sein de la doctrine. Au demeurant, il s'affirme progressivement comme une règle d'application directe et autonome dans des contentieux qui se rapportent à des décisions publiques prises dans un contexte d'incertitude scientifique. Cependant, il n'est pas devenu un "principe de droit" pouvant faire partie de l'arsenal juridique et auquel les Cours et tribunaux pourraient faire appel plus régulièrement pour sanctionner des comportements aux conséquences préjudiciables. Pourtant, il ne diffère pas fondamentalement des règles ordinaires de la responsabilité civile et bien évidemment de l'industrie de l'assurance.

Même s'il apparaît que le principe est mal utilisé, il constitue aujourd'hui un enjeu considérable de l'amélioration des assurances. Il n'en reste pas moins que la collectivité sera appelée à fixer de nouvelles règles relatives à la nature et au degré de risque acceptable au cas par cas. Néanmoins la réussite de ces efforts dépendra de la clarification de la contribution de la responsabilité civile au raisonnement en matière de précaution. "C'est en réalité un nouveau processus de décision qu'il faut inventer", pour utiliser les termes de Engel (1995).

Pour établir des recommandations des politiques à suivre au niveau national et international, l'arbitrage assurance, ex-post/principe de précaution/ex-ante qui a été mis en évidence dans cet article, doit être replacé dans une problématique plus large incluant notamment d'autres principes de «bon gouvernement» applicables au choix technologiques (Latour, 2000). Ces principes invitent à évoluer vers l'établissement de règles codifiées qui prescriront une procédure d'alerte ex-ante pour devancer à temps les effets des risques technologiques majeurs. Selon Baverez (2011), il faut mutualiser le risque ex-post, en recourant à d'autres règles telles que le principe de résilience. Cette nouvelle perspective devrait permettre de trancher la complexité apparente et enfin réfléchir sur d'autres bases assurantielles.

Nous pouvons conclure provisoirement que la question est loin d'être tranchée et que le débat reste ouvert en matière de responsabilité civile face aux incertitudes radicales. Il reste encore un long chemin à parcourir avant de trouver des solutions juridiques, économiques et sociales satisfaisantes. Raison pour laquelle, cet article peut être étendu dans plusieurs directions. Cette extension fera prochainement l'objet de notre réflexion. 


\section{BIBLIOGRAPHIE}

[1] Abenhaim, L. (1999). Nouveaux enjeux de santé publique: en revenir au paradigme du risque. Revue française des Affaires sociales, 53 (1), $31-44$.

[2] Audouy, L. (2015). Le principe de subsidiarité au sens du droit de la Convention Européenne des Droits de l'Homme (Mémoire de thèse, Université de Montpellier, France). Repéré à https://tel.archives-ouvertes.fr/ tel-01369159/document

[3] Barrieu, P. et Sinclair-Desgagné, B. (2003). The paradox of precaution. Cirano working papers, 63. Repéré à https://www.researchgate.net/ publication/5004738_The_Paradox_of_Precaution

[4] Baverez, N. (2011). De la tragédie du Japon au siècle des risques. Repéré à https://www.lepoint.fr/editos-du-point/nicolas-baverez/de-latragedie-du-japon-au-siecle-des-risques-22-03-2011-1312194_73.php\# $\mathrm{xtmc}=$ de-la-tragedie-du-japon-au-siecle-des-risques\&xtnp $=1 \& \mathrm{xtcr}=1$

[5] Bernard de Raymond, A. et Tétart, G. (2010). Le mouvement des Faucheurs Volontaires d'OGM. La désobéissance civique comme expérimentation de la citoyenneté. Journées Sociologues INRA. Repéré à https:// halshs.archives-ouvertes.fr/halshs-00617045/document

[6] Camproux-Duffrene,M-P. et Muller-Curzydlo, A. (2011). Chronique de droit privé de l'environnement, civil et commercial (2009-2011). Revue juridique de l'Environnement, 3, 365-392.

[7] Babisuaux, C., Le Déaut, J-Y., Sicard, D. et Testard, J. (2003). Plantes transgéniques: l'expérimentation est-elle acceptable? Paris, France: La documentation française.

[8] Daemen, G. et Georgelaan, L. (2013). Le principe de précaution. Journal D.A.S, 1-3.

[9] Dufumier, A. (2017). Les nuisances de la très haute tension reconnues. Repéré à http://www.lafranceagricole.fr/actualites/elevage/elevage-lesnuisances-de-la-tres-haute-tension-reconnues-1,1,1276102109.html

[10] Dumas, A. (2019). Les catastrophes naturelles tapent dans les profits des assureurs. Repéré à https://www.novethic.fr/actualite/finance-durable/ isr-rse/les-catastrophes-naturelles-tapent-dans-les-profits-des-assureurs146977.html

[11] Dumont, J-P. (2011). Gestion des risques des compagnies d'assurance: une revue de la littérature récente. Assurances et gestion des risques, 79 (1-2), 43-81. 
[12] Engel, L. (1995). La responsabilité en crise. Paris, France: Hachette.

[13] Ewald, A. C. (2012). Collateral consequences in the America States. Social science quarterly, 93 (1), 211-247.

[14] Fantoni-Quinton, S. et Saison-Demars, J. (2016). : Le principe de précaution face à l'incertitude scientifique: l'émergence d'une responsabilité spécifique dans le champ sanitaire. Cahier de recherche, Université de Lille2. Repéré à http://www.gip-recherche-justice.fr/publication/view/ le-principe-de-precaution-face-a-lincertitude-scientifique-lemergencedune-responsabilite-specifique-dans-le-champ-sanitaire/

[15] Florin, P. (2004). L'assurance confrontée au principe de l'assurance. Risques, les cahiers de l'assurance, 57, 106-108. Repéré à https://www. revue-risques.fr/wp-content/uploads/2019/04/Risques-057_Web.pdf

[16] Forest, G. (2011). Responsabilité, principe de précaution et causalité. Repéré à https://www.dalloz-actualite.fr/essentiel/responsabilite-principede-precaution-et-causalite\#.XO6IdRZKhqM .

[17] Giudicelli-Delage, G., Manacorda, S. et d'Ambrosio, L. (2016). Dynamiques normatives du principe de précaution et métamorphoses de la responsabilité juridique. Recherche réalisée avec le soutien de la Mission de Recherche Droit et Justice. Repéré à https://halshs.archives-ouvertes. fr/halshs-01480591/document

[18] Goasguen, C. (2011). Le principe de précaution à l'étude. Rapport de l'assemblée nationale et du comité d'évaluation et de contrôle des politiques publiques. France.

[19] Godard, O. (2000). Le principe de précaution: s'éloigner du positivisme scientifique pour civiliser les risques. Science et société, 294, 14.

[20] Gollier, G. et Treich, N. (2014). Les approches économiques de la précaution: présentation et discussion critique. Natures Sciences Sociétés, 22, 85-92.

[21] Ha Duong, M. (2005). Modèles de précaution en économie: introduction aux probabilités imprécises (Mémoire d'habilitation à diriger les recherches, Université de Paris I Panthéon-Sorbonne, Paris, France). Repéré à https://tel.archives-ouvertes.fr/file/index/docid/52919/filename/ HaDuong-20051223-ModelesDePrecautionEnEconomieIntroduction AuxProbabilitesImprecises.pdf

[22] Hajtaieb, N. et Yanhui, E. W. (2015). Le besoin de réassurance: de la psychologie au comportement du consommateur; Une investigation théorique appliquée au cas de la consommation alimentaire moderne. $L a$ Revue Gestion et Organisation, 7, 1-8. 
[23] Hémon, J. (2015). Le rôle de la Cour de justice de l'Union européenne dans le processus de constitution et d'évolution du marché intérieur des marchandises (Thèse de doctorat, Université de Rennes 1, Rennes, France). Repéré à https://ged.univ-rennes1.fr/nuxeo/site/esupversions/ 2b754560-4de6-4396-a3e7-5e6bb0048ec9?inline

[24] Hunyadi, M. (2003). Pourquoi avons-nous besoin du raisonnement de précaution? Esprit, 8-9, 139-162.

[25] Joiner, T. E., Metalsky, G. I., Katz, J. et Beach, S. R. H. (1999). Depression and Excessive Reassurance- seeking. Psychological Inquiry, 10 (4), 269-278.

[26] Keynes, J. M. (1936). The general theory of employment, interest and money. London: Macmillan.

[27] Lagadec, P. (1988). Le risque technologique majeur. Problèmes économiques et sociaux, 591, 47.

[28] Latour, B. (2000). Du principe de précaution au principe de bon gouvernement. Études, 393-4, 339-346.

[29] Marie, A. (2014). Les compagnies d'assurance refusent d'assurer les cultures d'OGM. Repéré à https://reporterre.net/Les-compagnies-dassurance

[30] Martineau, A.-C. (2013). Une analyse critique du débat sur la fragmentation du droit international (Thèse de doctorat, Université PanthéonSorbonne, Paris, France). Repéré à https://tel.archives-ouvertes.fr/ tel-01259489/document

[31] Nordhaus, W. D. (1994). Managing the Global Commons. Cambridge (MA): The MIT Press.

[32] November, A. et November, V. (2004). Risque, assurance et irréversibilité, Revue européenne des sciences sociales, XLII-130, 161-179. Repéré à http://journals.openedition.org/ress/475

[33] OCDE. (2003). Emerging Systemic Risks: A New Frontier? Presentation by Schieb P.-A. at the - Conference: Risikogesellschaft Wohin? OCDE International Futures Programme, Suisse.

[34] Searalini, G.-E. (2003). Génétiquement incorrect. Paris, France: Flammarion.

[35] Simonnet, C. (2015). La gestion des risques portés par le client en banque et assurance: Comportements et éthique des acteurs (Thèse soutenue à l'école doctorale management et société, Conservatoire National des Arts et Métiers). Repéré à https://tel.archives-ouvertes.fr/tel-01344414/ document 
[36] Thourot, P. (2016). La réassurance: quel rôle dans le développement économique dans les pays émergents? Techniques Financières et Développement, 1 (122), 15-21.

[37] Triech, N. (2001). Le principe de précaution est-il économiquement acceptable. INRA Sciences sociales, 6/00, 1-4. Repéré à https://tindcustomer-agecon.s3.amazonaws.com/c8bf5ef2-b1f5-488b-ad84b024be0ace82?response-content-disposition=inline $\% 3 \mathrm{~B} \% 20$ filename $\% 2 \mathrm{~A} \% 3 \mathrm{DUTF}-8 \% 27 \% 27$ iss00-6.pdf\&response-content-type=application \%2Fpdf\&AWSAccessKeyId=AKIAXL7W7Q3XHXDVDQYS\&Expires $=1558977439 \&$ Signature $=\mathrm{ds} 4 \mathrm{Kw}$ GiHFq6sjdn2ey2PYOU0v7Q\%3D

[38] Triech, N. (2005). L'analyse coût-bénéfice de la prévention des risques. Université de Toulouse, LERNA-INRA, 1-52. Repéré à http:// www2.toulouse.inra.fr/lerna/treich/ACB03.pdf

[39] Weinstock, L. M. et Whisman, M. A. (2006). Rumination and excessive reassurance-seeking in depression : a cognitive-interpersonal integration. Cognitive Therapy and Research, 30 (3), 333-342.

[40] Yombatina, B. (2010). Le principe de précaution en droit de l'environnement: règle juridique obligatoire ou simple principe politique destiné à porter et conséquences dans ordres juridiques international et national. Repéré à http://www.dhdi.free.fr/recherches/environnement/ articles/sitackprecaution.htm

\section{NOTES}

1 Faculté des sciences juridiques économiques et sociales, Université Cadi Ayyad, Marrakech. Courriel: Elghezaoui2000@yahoo.fr.

2 La prévention vise les risques avérés, ceux dont l'existence est démontrée ou connue empiriquement sans toutefois qu'on puisse en estimer la fréquence d'occurrence. Exemple: le risque nucléaire. L'incertitude ne porte pas sur le risque, mais sur sa probabilité de réalisation. L'absence de probabilités rend le risque inassurable par l'industrie classique de l'assurance.

3 La précaution se distingue de la protection contre les dangers. La différence réside dans l'identification du risque.

4 Voir par exemple la formulation dans la Déclaration de Rio (Conférence des Nations-Unies sur l'Environnement et le Développement, 1992, dans son principe 15) : "En cas de risques de dommages graves ou irréversibles, l'absence de certitude scientifique ne doit pas servir de prétexte pour remettre à plus tard l'adoption de mesures économiquement efficaces visant à prévenir la dégradation de l'environnement».

5 II ne s'agit pas de la première fois que l'industrie de l'assurance se trouve devant le dilemme de devoir assurer une activité dont les risques sont inconnus. Elle connait notamment la notion de « risque de développement» qui répond précisément à la situation de devoir envisager des risques qui ne sont pas connus au moment de la mise des produits sur le marché.

6 Dans le domaine des assurances, la responsabilité se définit comme l'obligation de réparer le dommage que l'on a causé.

7 Tel que rapporté dans https://www.cci.fr/universite/universite11/documents/principedeprecaution. 